\title{
Fluorescence lifetime imaging of fluorescent proteins as an effective quantitative tool for noninvasive study of intracellular processes
}

\author{
Svitlana M. Levchenko*, Artem Pliss ${ }^{\dagger}$ and Junle $\mathrm{Qu}^{* *}$ \\ *Key Laboratory of Optoelectronic Devices \\ and Systems of Guangdong Province \\ College of Optoelectronic Engineering, Shenzhen University \\ Shenzhen, Guangdong Province 518060, P. R. China \\ ${ }^{\dagger}$ Institute for Lasers, Photonics and Biophotonics \\ University at Buffalo, State University of New York \\ Buffalo, NY 14260-3000, USA \\ ثjlqu@szu.edu.cn
}

Received 16 April 2017

Accepted 7 June 2017

Published 19 July 2017

\begin{abstract}
Fluorescence lifetime imaging (FLIM) is an effective noninvasive bioanalytical tool based on measuring fluorescent lifetime of fluorophores. A growing number of FLIM studies utilizes genetically engineered fluorescent proteins targeted to specific subcellular structures to probe local molecular environment, which opens new directions in cell science. This paper highlights the unconventional applications of FLIM for studies of molecular processes in diverse organelles of live cultured cells.
\end{abstract}

Keywords: Fluorescence lifetime imaging; fluorescent proteins; bioimaging; intracellular processes.

\section{Introduction}

Over the decades, fluorescence microscopy has been a dominant tool for visualization of complex cellular structure, mapping of biomolecules and studies of various cellular processes. ${ }^{1,2}$ The ongoing development of fluorescence spectroscopy technology has enabled for noninvasive acquisition of spectroscopic information from a single cell with ever increasing accuracy, speed and sensitivity. ${ }^{3,4}$ At the same time, a significant progress was accomplished in genetic engineering of fluorescent proteins (FPs). Current technology enables a facile fusion of FPs to diverse cellular proteins, which further revolutionized studies of molecular structure in live cultured cells.

\$Corresponding author.

This is an Open Access article published by World Scientific Publishing Company. It is distributed under the terms of the Creative Commons Attribution 4.0 (CC-BY) License. Further distribution of this work is permitted, provided the original work is properly cited. 
The first FP — jellyfish-derived green fluorescent protein (GFP) - was discovered by Shimomura in 1960s. ${ }^{5}$ Afterwards, GFP gene was cloned by Prasher ${ }^{6}$ and in 1994, it was reported that GFP can be expressed both in bacteria and multicellular organisms such as E. coli and C. elegans. ${ }^{7,8}$ These findings made it possible the widespread applications of GFP as a marker for visualization of gene expression in living organisms. Later structural studies revealed that GFP displays complex $\beta$-barrel structure with the chromophore located in the core of the protein. ${ }^{9}$ This exceptional structure allows for production of a number of mutants with different spectral characteristics via modifications of chromophore and/or its local environment. ${ }^{8,10-12}$ Using this approach, a number of GFP-derived mutants (e.g., EGFP, YFP, CFP, BFP) and other $m \mathrm{RFP} 1-$ derived FPs (e.g., $t d$ Tomato, $m$ Orange, $m$ Cherry, etc.) with improved characteristics (e.g., enhanced photostability and brightness) were produced, characterized and applied for cell biology studies. Moreover, the absorption/emission spectra of up-to-date generated mutants essentially cover entire visible spectrum. ${ }^{8,13-19}$ The exceptional properties of FPs made them indispensable for live cell bioimaging. ${ }^{20-22}$

Currently, the synthesis of FPs with excitation/ emission in the near-infrared region (NIR) has been actively pursued. New family of NIR-FPs offers numerous advantages for imaging in live cells and tissues, including low phototoxicity, deep penetration through biological material in the biological transparency window $(\sim 650-950 \mathrm{~nm})$, as well as potentially higher detection sensitivity due to low light scattering and reduced autofluorescence. ${ }^{23-25}$ Although few efficient NIR FPs have been synthesized to date, and their brightness lags behind conventional labels, some newly synthesized FPs look very promising. For instance, a family of phytochrome-based NIR FPs ( $i$ RFP) has already been validated for imaging in live cells, tissues and even whole animals. ${ }^{23,24,26}$

It is worth to note that FP tags can alter the three-dimensional (3D) conformation of the target protein and/or affect their functions. ${ }^{27}$ However, such cases are rare, and could be further reduced by genetic modifications of FP fusions. ${ }^{28}$

In the recent two decades, FPs have been increasingly utilized as probes for fluorescence lifetime imaging (FLIM). For instance, proteins like GFP and its mutants can also be tracked and distinguished by their fluorescence lifetime, the average time between its excitation and its return to the ground state. ${ }^{1}$ There are two common techniques for measuring fluorescence lifetime: the timedomain (TD) and frequency-domain (FD) methods. TD requires a pulsed light-source (much shorter than the decay time of the sample), while FD requires a sinusoidal intensity-modulated light (its reciprocal frequency should be comparable to the reciprocal of decay time). ${ }^{1,32}$ In TD technique, the fluorescence decay function is directly recorded, while in FD, the information about fluorescence decay is obtained from the difference between the modulation degree and a phase shift of the sample fluorescence and the excitation. The TD measurements are often performed using time-correlated single-photon counting (TCSPC) technique and the fluorescence decay is acquired in each pixel of the image.

In combination with modern imaging setups, FLIM paves the way for new discoveries in biomedical science. ${ }^{4,29-31}$ Remarkably, in comparison with fluorescence intensity-based techniques, fluorescence lifetime measurements are independent of changes in probe concentration, excitation intensity fluctuation and photobleaching, thus representing a more robust and reproducible bioanalytical tool. ${ }^{1,4}$ There are a number of reviews and quite a few books that contain detailed description of basic principles of FLIM, as well as recent technical achievements and data analysis that allow to perform lifetime measurements with high accuracy in various samples, including live cells and tissues. ${ }^{4,29,32-34}$ Therefore, this paper does not contain this kind of information and is mainly focused on FP applications for studies of cellular molecular processes by FLIM.

\subsection{High resolution protein trafficking}

Achievements in FLIM methods as well as the engineering of new FPs with enhanced spectral properties open up new horizons for studies of complex cellular structure with high accuracy. For instance, improved mutant of YFP, $m$ Citrine protein, was used as a lifetime-based sensor for probing protein localization as well as cytosolic protein PINK1 displacement between the mitochondrial microcompartments. ${ }^{35}$ Remarkably, because of small diameter of mitochondria, mapping of protein 
localization in its microcompartments formerly was not achievable by conventional fluorescence microscopy. However, it was found that localization in inner and outer membrane can be identified by the difference in lifetime of $m$ Citrine tag. This difference was due to inverse correlation between fluorescence lifetime of fluorophores and local refractive index. ${ }^{36}$ Since the refractive index of membranes is significantly higher than that of mitochondrial matrix, the fluorescence lifetime of membrane-bound FP-fusions is shorter than that in the mitochondrial matrix, which enabled for mapping of the FPs localization in mitochondria with a sub-diffraction resolution. Moreover, it was demonstrated that FLIM approach is sensitive enough even for protein trafficking within mitochondrial microcompartments. ${ }^{35}$

Furthermore, the potential of FLIM techniques for multiplex imaging was demonstrated. ${ }^{37}$ The algorithm for signal differentiation from up to five fluorophores with similar fluorescence emission spectra was introduced recently. ${ }^{37}$ In that study, five fluorescent probes, including EGFP, ATTO-488, ATTO 488 azide, Alexa Fluor 488 and ATTO490LS were used in the cultured cells for labeling of nuclear envelope, cytoskeleton, nascent DNA, Golgi Apparatus and the nucleoli, respectively. All the probes were excited by the same $485 \mathrm{~nm}$ excitation wavelength. Next, the pattern-matching algorithm was applied for selective identification of each fluorophore, thus providing a concept for highly multiplex imaging
(Fig. 1). Moreover, the ability to visualize up to nine targets using spectrally resolved FLIM microscopy (sFLIM) was recently demonstrated. ${ }^{37}$

In another study, FLIM was applied for investigating spatial distribution of nucleocapsid protein (NCp7) of the Human immunodeficiency virus type 1 (HIV-1) in HeLa cells. ${ }^{38}$ In this study, NCp7 was tethered with EGFP and the cellular RNA was labeled with Sytox Orange, to probe the FRET between both fluorophores. It was shown that the main sites for NCp7-EGFP localization are cytoplasm and the nucleoli, where it binds to cellular or ribosomal RNAs. Based on these data, the authors suggested that NCp7 molecules may primarily bind to ribosomes after releasing in the infected cells. ${ }^{38}$

\section{2. $\quad$ Monitoring intracellular $\boldsymbol{p H}$ level, temperature and protein concentration}

Due to the specific location of fluorophore inside the proteins $\beta$-barrel, the spectral properties of these types of FPs can be modulated through their interactions with the environment. This feature of FPs can be used for sensing changes in their environment such as intracellular $\mathrm{pH},{ }^{39-41}$ temperature ${ }^{42}$ or protein concentration. ${ }^{43,44}$ As value of these parameters in specific cellular compartments plays an important role in regulation of many intracellular processes, the reliable, accurate and noninvasive methods for (a)

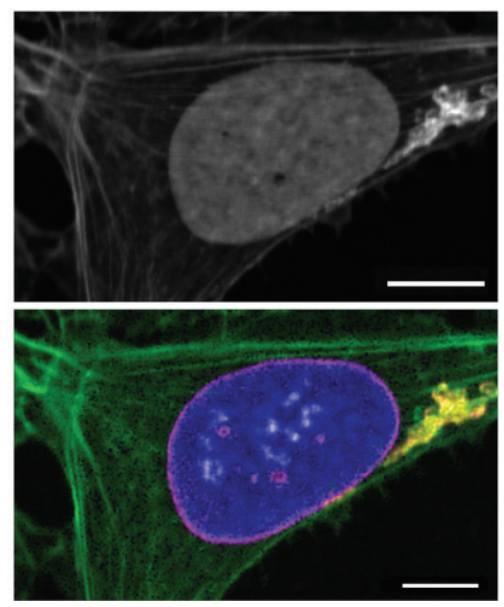

(c)

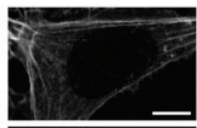

(d)

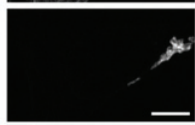

Fluorescent labels:

ATTO 488

Alexa Fluor 488

(e)

(f)

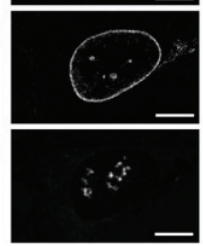

\section{ATTO 488 azide}

\section{EGFP}

\section{ATTO 490LS}

Source: Reprinted by permission from Macmillan Publisher Ltd: Nature Methods, ${ }^{37}$ Copyright 2016.

Fig. 1. Multitarget images of different cellular structures labeled with five fluorescent probes with similar fluorescent properties: (a) Total fluorescence intensity image of an U2OS cell. (b) sFLIM composite image showing (green, c) $F$-actin, (yellow, d) Golgi, (blue, e) nascent DNA inside the nucleus, (magenta, f) nuclear envelope, and nucleoli (white, g). Scale bars, $10 \mu \mathrm{m}$. 
determination of intracellular parameters are highly desirable in molecular biology and biomedicine. In this regard, genetically encoded FPs-based sensors have a big advantage, as they can be targeted to almost any cellular compartment without loading procedure that is required for conventional dyes. First, it was found that absorbance and fluorescence of GFP mutants strongly depend on $\mathrm{pH}$ value both in vitro as well as in intracellular compartments of live cells. ${ }^{39,40}$ Probable reason could be that $\mathrm{pH}$ can shift equilibrium between two existing GFP ground states, which has different spectroscopic characteristics. ${ }^{39}$ For instance, the response of fluorescence of GFP mutant GFP-F64L/S65T to $\mathrm{pH}$ changes is rapid and reversible in the range between 6.5 and down to $5.0 .{ }^{39}$ Notably, there are plenty of dyes and some FPs with $\mathrm{pH}$ sensitive fluorescence intensity that can be used for intracellular $\mathrm{pH}$ evaluation by conventional fluorescence microscopy. ${ }^{45}$ However, one of the major drawbacks of fluorescence intensitybased $\mathrm{pH}$ sensors is that obtained data are not always clear, as the fluorescence intensity can be affected not only by $\mathrm{pH}$ changes, but also by fluorophore concentration fluctuations (e.g., FPs expression level which is troublesome to control). Application of FLIM technique allows for overcoming this limitation, making it more favorable for intracellular pH mapping. There are a few reports where FLIM was used as a nondestructive and efficient method for $\mathrm{pH}$ distribution mapping, and monitoring of $\mathrm{pH}$ dynamics in different cellular organelles via changes in FPs lifetime values. ${ }^{41,46-48}$ For instance, lifetime of engineered red fluorescent protein pHRed showed a high sensitivity to intracellular $\mathrm{pH}$ in live Neuro2A cells. ${ }^{41}$ The lifetime of pHRed notably increased upon changing of $\mathrm{pH}$ value from 5 to 8 . Magnitude of observed changes in lifetime was about $\sim 0.4$ ns that can be effectively detected by FLIM. Furthermore, the correlation between fluorescence lifetime of enhanced yellow fluorescent protein (EYFP) and local $\mathrm{pH}$ ranging from 4.5 to 9, both in buffer solution and in HeLa cells with different excitation wavelengths was studied (Fig. 2). ${ }^{46}$ It was shown that the sensitivity of EYFP lifetime in buffer solution depends on excitation wavelength. The best results for measuring intracellular $\mathrm{pH}$ by the FLIM of EYFP in HeLa cell line were obtained with excitation at $440 \mathrm{~nm}$ in the $\mathrm{pH}$ range of $4.5-6.0$, which is essential for acidic organelles such as lysosomes. ${ }^{46}$

Another study demonstrated that intracellular lifetime of widely used enhanced cyan fluorescent protein (eCFP) also exhibits large changes

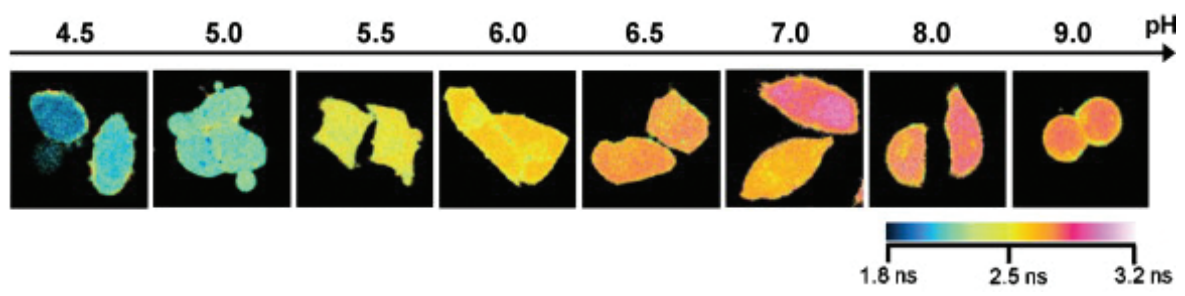

(a)

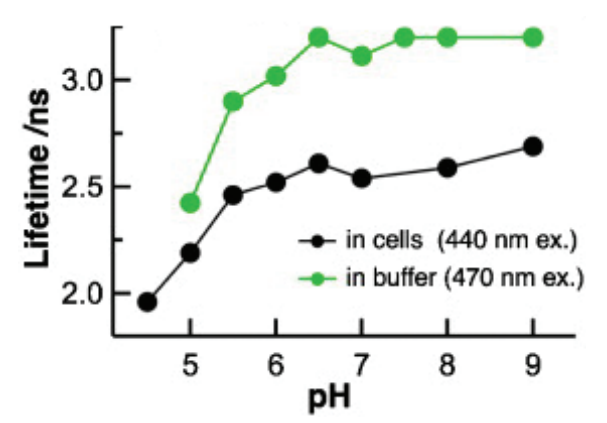

(b)

Source: Reprinted from Ref. 46. Copyright 2012, with permission from Elsevier. ${ }^{46}$

Fig. 2. (a) The correlation between fluorescence lifetime of EYFP expressed in HeLa cells and intracellular pH. (b) Dependence of average EYFP fluorescence lifetime on $\mathrm{pH}$ in cells and buffer solution. 
(around $32 \%$ ) in the $\mathrm{pH}$ range from 5.0 to 7.0 , which allows to perform $\mathrm{pH}$ measurements in intracellular compartment with high accuracy around $0.2 \mathrm{pH}$ unit. ${ }^{49}$ Notably, eCFP is suitable for $\mathrm{pH}$ measuring at acidic $\mathrm{pH}$ values (from 5.0 to 7.0 ) at the range of physiologically relevant temperature $20-37^{\circ} \mathrm{C}$. Moreover, the possibility to use it for tracking dynamical changes in acidic secretory granules of PC12 cells was demonstrated. ${ }^{49}$ Enhanced GFP (eGFP) was also proposed as a lifetime-based $\mathrm{pH}$ sensor for evaluation in the cytoplasm or mitochondria of Chinese hamster ovary cells. ${ }^{47}$ The lifetime of eGFP was used as a parameter for the quantitative analysis of $\mathrm{pH}$ in cells under excitation at $405 \mathrm{~nm}$. It was demonstrated that $\mathrm{pH}$ in the mitochondrial matrix is around 7.8 and it is slightly higher than the $7.0 \mathrm{pH}$ value in cytoplasm.

Another interesting application for FPs was suggested after the discovery of the temperature dependence of GFP fluorescence signal intensity, fluorescence anisotropy and fluorescence lifetime. ${ }^{42,50-53}$

The correlation between the signal intensity and temperature was exploited in several studies. It was demonstrated that fluorescence intensity of certain FPs exhibit thermal sensitivity, thus enabling intracellular temperature sensing. Genetically encoded GFP-based thermosensor, named tsGFPs, was applied for monitoring the temperature of the plasma membrane, mitochondria and endoplasmic reticulum in living cells. ${ }^{51}$ Temperature-sensitive conformational transformations in tsGFP in turn caused changes in the intensity ratio between two characteristics emission peaks of tsGFP at $400 \mathrm{~nm}$ and $480 \mathrm{~nm}$. As a result, the thermal heterogeneity in mitochondria was detected. Furthermore, the dependence between temperature and membrane potential was demonstrated. ${ }^{51}$ Another group developed ratiometric thermometer (gTEMP) by using two FPs with different temperature sensitivity. ${ }^{52}$ gTEMP enabled fast tracking of the temperature changes in the range of $5-50^{\circ} \mathrm{C}$ with temporal resolution of $50 \mathrm{~ms}$. Using this thermometer the authors observed the temperature distribution in the cytoplasm and nucleus in cells. Moreover, this approach has been used for monitoring heat generation in mitochondria matrix in a single living cell. In comparison, most of conventional FPs-based thermometry methods exploit fluorescence intensity changes for monitoring temperature distribution inside the single cell. The inherent disadvantages of these methods include susceptibility to photobleaching as well as fluctuations of the excitation light intensity and chromophore concentration. In this regard, the temperature monitoring methodbased either FPs or polymeric structures with temperature dependent fluorescence lifetime offer significant advantages. ${ }^{42,54,55}$

Recently, another advanced method for temperature mapping in single cells based on characteristic properties of green and red fluorescent protein (GFP and $m$ RFP) was developed. ${ }^{42,53}$ In these studies, it was shown that the fluorescence intensity and fluorescence lifetime of FPs serve as accurate temperature evaluation. A temperature accuracy of about $0.4^{\circ} \mathrm{C}$ was achieved at the single cell level. The temperature differences in various organelles were detected. Another group demonstrated that the lifetime of ER thermo yellow and $m$ Cherry exhibits linear dependence on temperature in the range of $24-40^{\circ} \mathrm{C}$ and can be used as organelle thermometer. Using FLIM of living myotubes loaded with ER thermo yellow and cytosolic $m$ Cherry, the quantitative imaging of heat production induced by $\mathrm{Ca}^{2+}$ in myotubes was performed. ${ }^{54}$ Advances in lifetime-based technique and engineering of the FPs with desired properties may lead to the development of intracellular thermosensors with better parameters (e.g., higher sensitivity, faster response, broader temperature range, etc.).

Furthermore, from fluorescence lifetime measurements of GFP, the information about local refractive index, intracellular concentration of macromolecules and cell cycle progression can be acquired.

In one study, it was shown that local refractive index evaluated from GFP lifetime value in live cells can serve as a tool for probing local environment in the immunological synapse. ${ }^{56}$ The correlation between inverse fluorescence lifetime of GFP-tagged protein in the membrane and corresponding refractive index was reported. It was suggested that the changes in lipid or protein concentration in local cellular environment may result in variations of local refractive index of GFP-tagged protein. ${ }^{56}$

In another study, the dependence of fluorescence lifetime on local refractive index was utilized for the measurement and monitoring of biomolecular concentrations in specific subcellular compartments. $^{43,44}$ First, the fluorescence lifetime of GFP and $t d$ Tomato was employed for sensing of 


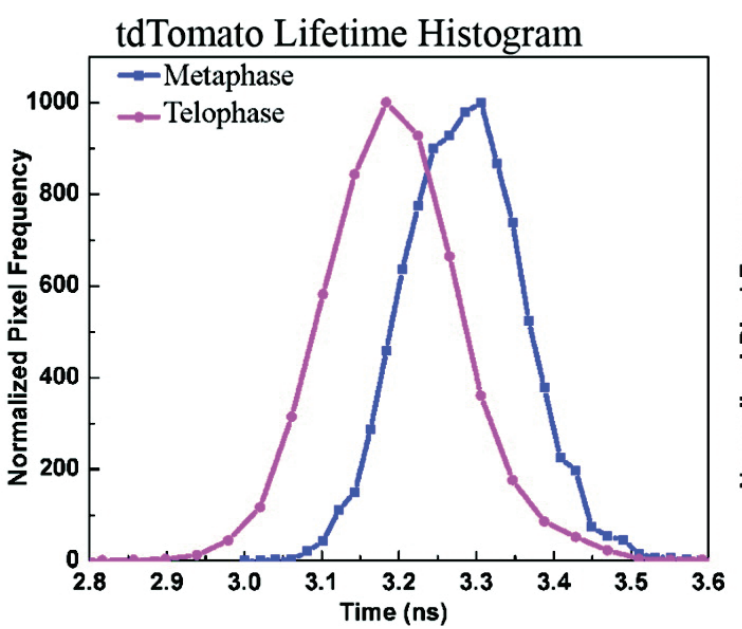

(a)

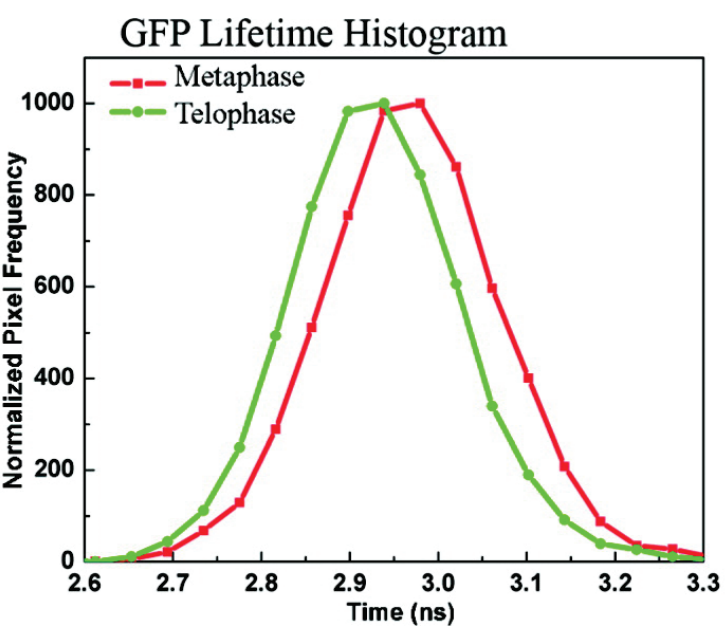

(b)

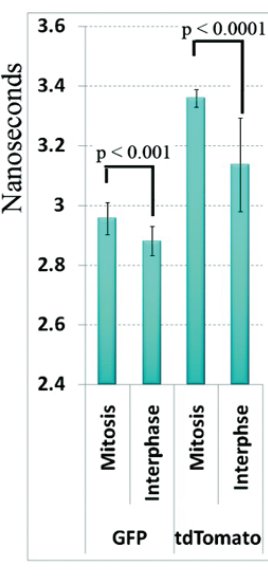

(c)

Source: Reprinted with permission from Ref. 43. Copyright 2012 American Chemical Society. ${ }^{43}$

Fig. 3. Alteration of fluorescence lifetime distributions of (a) tdTomato and (b) GFP in cells during the transition from mitosis to interphase. (c) The statistical analysis of fluorescence lifetimes measured in interphase cells nuclei and in mitotic cells.

concentration changes in the nucleoplasm of HeLa cells during the cell cycle progression. ${ }^{43}$ It was found that fluorescence lifetime values of GFP and $t d$ Tomato are notably shortened during the transition from mitosis to interphase, evidently due to rapid increase in the refractive index during the cell division. The difference in average lifetime for GFP and $t d$ Tomato was $80 \mathrm{ps}$ and 220 ps correspondingly (Fig. 3).

In the following study, the intracellular refractive index was derived from FPs lifetime. Next, based on refractive index, the absolute protein concentrations in cellular environment were calculated. This approach was applied to measure and monitor the absolute concentrations of proteins into major structurefunction compartments of the cell nucleus: nucleoli and the nuclear speckles. In these nuclear compartments the average protein concentration was at $\sim 284 \mathrm{mg} / \mathrm{mL}$ and $\sim 150 \mathrm{mg} / \mathrm{mL}$, respectively. ${ }^{44}$

\section{Summary}

FLIM appeared recently as a noninvasive, very sensitive, informative and powerful method that has found a wide range of applications in cellular biology and medicine. Application of FPs as a molecular probe for FLIM gives access to the new layer of information that cannot be reached by conventional fluorescence microscopy. Using fluorescence lifetime of FPs instead of fluorescence intensity allows for the identification of protein localization, protein trafficking in specific organelle, probing and monitoring of changes in protein environment and consequently measuring pivotal intracellular parameters (e.g., pH, temperature, refractive index, overall protein concentration and/or viscosity) in single cell or cellular compartments without cell damaging. The synergic development of optical technology and molecular biology promises further breakthroughs in studies of cellular molecular structure and behavior.

\section{Acknowledgments}

Parts of this work were supported by the National Basic Research Program of China (2015CB352005); the National Natural Science Foundation of China (61525503/61378091/61620106016); Guangdong Natural Science Foundation Innovation Team (2014A030312008); Hong Kong, Macao and Taiwan cooperation innovation platform and major projects of international cooperation in Colleges and Universities in Guangdong Province (2015KGJHZ002); and Shenzhen Basic Research Project (JCYJ2015 0930104948169/JCYJ20160328144746940/GJHZ 20160226202139185).

\section{References}

1. J. R. Lakowicz, Principles of Fluorescence Spectroscopy, 3rd edition, Springer, New York (2006). 
2. A. Ettinger, T. Wittmann, "Fluorescence live cell imaging," Methods Cell. Biol. 123, 77-94 (2014).

3. J. C. Waters, T. Wittmann, "Quantitative imaging in cell biology: Preface," Methods Cell. Biol. 123, $\mathrm{xix}-\mathrm{xx}(2014)$.

4. W. Becker, Advanced Time-Correlated Single Photon Counting Techniques, Springer, Berlin (2005).

5. O. Shimomura, F. H. Johnson, Y. Saiga, "Extraction, purification and properties of aequorin, a bioluminescent protein from the luminous hydromedusan, Aequorea," J. Cell. Comp. Physiol. 59, 223-239 (1962).

6. D. C. Prasher, V. K. Eckenrode, W. W. Ward, F. G. Prendergast, M. J. Cormier, "Primary structure of the Aequorea victoria green-fluorescent protein," Gene 111, 229-233 (1992).

7. M. Chalfie, Y. Tu, G. Euskirchen, W. W. Ward, D. C. Prasher, "Green fluorescent protein as a marker for gene-expression," Science 263, 802-805 (1994).

8. M. Zimmer, "GFP: From jellyfish to the Nobel prize and beyond," Chem. Soc. Rev. 38, 2823-2832 (2009).

9. M. Ormo, A. B. Cubitt, K. Kallio, L. A. Gross, R. Y. Tsien, S. J. Remington, "Crystal structure of the Aequorea victoria green fluorescent protein," Science 273, 1392-1395 (1996).

10. R. Heim, D. C. Prasher, R. Y. Tsien, "Wavelength mutations and posttranslational autoxidation of green fluorescent protein," Proc. Natl. Acad. Sci. USA 91, 12501-12504 (1994).

11. R. Heim, A. B. Cubitt, R. Y. Tsien, "Improved green fluorescence," Nature 373, 663-664 (1995).

12. G. J. Kremers, S. G. Gilbert, P. J. Cranfill, M. W. Davidson, D. W. Piston, "Fluorescent proteins at a glance," J. Cell. Sci. 124, 157-160 (2011).

13. A. W. Scruggs, C. L. Flores, R. Wachter, N. W. Woodbury, "Development and characterization of green fluorescent protein mutants with altered lifetimes," Biochemistry 44, 13377-13384 (2005).

14. A. S. Mishin, F. V. Subach, I. V. Yampolsky, W. King, K. A. Lukyanov, V. V. Verkhusha, "The first mutant of the Aequorea victoria green fluorescent protein that forms a red chromophore," Biochemistry 47, 4666-4673 (2008).

15. M. V. Matz, A. F. Fradkov, Y. A. Labas, A. P. Savitsky, A. G. Zaraisky, M. L. Markelov, S. A. Lukyanov, "Fluorescent proteins from nonbioluminescent Anthozoa species," Nat. Biotechnol. 17, 969-973 (1999).

16. N. C. Shaner, R. E. Campbell, P. A. Steinbach, B. N. Giepmans, A. E. Palmer, R. Y. Tsien, "Improved monomeric red, orange and yellow fluorescent proteins derived from Discosoma sp. red fluorescent protein," Nat. Biotechnol. 22, 1567-1572 (2004).
17. R. Heim, R. Y. Tsien, "Engineering green fluorescent protein for improved brightness, longer wavelengths and fluorescence resonance energy transfer," Curr. Biol. 6, 178-182 (1996).

18. P. E. Konold, E. Yoon, J. Lee, S. L. Allen, P. P. Chapagain, B. S. Gerstman, C. K. Regmi, K. D. Piatkevich, V. V. Verkhusha, T. Joo, R. Jimenez, "Fluorescence from multiple chromophore hydrogenbonding states in the far-red protein TagRFP675," J. Phys. Chem. Lett. 7, 3046-3051 (2016).

19. D. S. Bindels, L. Haarbosch, L. van Weeren, M. Postma, K. E. Wiese, M. Mastop, S. Aumonier, G. Gotthard, A. Royant, M. A. Hink, T. W. Gadella, Jr., "mScarlet: A bright monomeric red fluorescent protein for cellular imaging," Nat. Methods 14, 53-56 (2017).

20. C. P. Toseland, "Fluorescent labeling and modification of proteins," J. Chem. Biol. 6, 85-95 (2013).

21. M. Modesti, "Fluorescent labeling of proteins," Methods Mol. Biol. 783, 101-120 (2011).

22. K. M. Dean, A. E. Palmer, "Advances in fluorescence labeling strategies for dynamic cellular imaging," Nat. Chem. Biol. 10, 512-523 (2014).

23. G. S. Filonov, K. D. Piatkevich, L. M. Ting, J. Zhang, K. Kim, V. V. Verkhusha, "Bright and stable near-infrared fluorescent protein for in vivo imaging," Nat. Biotechnol. 29, 757-761 (2011).

24. K. G. Chernov, T. A. Redchuk, E. S. Omelina, V. V. Verkhusha, "Near-infrared fluorescent proteins, biosensors, and optogenetic tools engineered from phytochromes," Chem. Rev. 117, 6423-6446 (2017).

25. E. Hemmer, A. Benayas, F. Legare, F. Vetrone, "Exploiting the biological windows: Current perspectives on fluorescent bioprobes emitting above 1000 nm," Nanoscale Horiz. 1, 168-184 (2016).

26. D. M. Shcherbakova, V. V. Verkhusha, "Nearinfrared fluorescent proteins for multicolor in vivo imaging," Nat. Methods 10, 751-754 (2013).

27. G. Crivat, J. W. Taraska, "Imaging proteins inside cells with fluorescent tags," Trends Biotechnol. 30, 8-16 (2012).

28. E. Snapp, "Design and use of fluorescent fusion proteins in cell biology," Curr. Protoc. Cell Biol. 21, 21-24 (2005).

29. R. Ebrecht, C. Don Paul, F. S. Wouters, "Fluorescence lifetime imaging microscopy in the medical sciences," Protoplasma 251, 293-305 (2014).

30. J. A. Levitt, D. R. Matthews, S. M. Ameer-Beg, K. Suhling, "Fluorescence lifetime and polarizationresolved imaging in cell biology," Curr. Opin. Biotechnol. 20, 28-36 (2009).

31. A. Periasamy, R. M. Clegg, FLIM Microscopy in Biology and Medicine, Taylor \& Francis, Boca Raton (2010). 
32. K. Suhling, L. M. Hirvonen, J. A. Levitt, P.-H. Chung, C. Tregidgo, A. Le Marois, D. A. Rusakov, K. Zheng, S. Ameer-Beg, S. Poland, "Fluorescence lifetime imaging (FLIM): Basic concepts and some recent developments," Med. Photonics 27, 3-40 (2015).

33. M. Y. Berezin, S. Achilefu, "Fluorescence lifetime measurements and biological imaging," Chem. Rev. 110, 2641-2684 (2010).

34. A. Le Marois, S. Labouesse, K. Suhling, R. Heintzmann, "Noise-Corrected Principal Component Analysis of fluorescence lifetime imaging data," J. Biophoton. (2016).

35. A. C. Sohnel, W. Kohl, I. Gregor, J. Enderlein, B. Rieger, K. B. Busch, "Probing of protein localization and shuttling in mitochondrial microcompartments by FLIM with sub-diffraction resolution," Biochim. Biophys. Acta 1857, 1290-1299 (2016).

36. K. Suhling, J. Siegel, D. Phillips, P. M. French, S. Leveque-Fort, S. E. Webb, D. M. Davis, "Imaging the environment of green fluorescent protein," Biophys. J. 83, 3589-3595 (2002).

37. T. Niehorster, A. Loschberger, I. Gregor, B. Kramer, H. J. Rahn, M. Patting, F. Koberling, J. Enderlein, M. Sauer, "Multi-target spectrally resolved fluorescence lifetime imaging microscopy," Nat. Methods 13, 257-262 (2016).

38. H. Anton, N. Taha, E. Boutant, L. Richert, H. Khatter, B. Klaholz, P. Ronde, E. Real, H. de Rocquigny, Y. Mely, "Investigating the cellular distribution and interactions of HIV-1 nucleocapsid protein by quantitative fluorescence microscopy," PLoS One 10, e0116921 (2015).

39. M. Kneen, J. Farinas, Y. Li, A. S. Verkman, "Green fluorescent protein as a noninvasive intracellular $\mathrm{pH}$ indicator," Biophys. J. 74, 1591-1599 (1998).

40. M. F. Abad, G. Di Benedetto, P. J. Magalhaes, L. Filippin, T. Pozzan, "Mitochondrial pH monitored by a new engineered green fluorescent protein mutant," J. Biol. Chem. 279, 11521-11529 (2004).

41. M. Tantama, Y. P. Hung, G. Yellen, "Imaging intracellular $\mathrm{pH}$ in live cells with a genetically encoded red fluorescent protein sensor," J. Am. Chem. Soc. 133, 10034-10037 (2011).

42. J. S. Donner, S. A. Thompson, M. P. Kreuzer, G. Baffou, R. Quidant, "Mapping intracellular temperature using green fluorescent protein," Nano Lett. 12, 2107-2111 (2012).

43. A. Pliss, L. Zhao, T. Y. Ohulchanskyy, J. Qu, P. N. Prasad, "Fluorescence lifetime of fluorescent proteins as an intracellular environment probe sensing the cell cycle progression," ACS Chem. Biol. 7, 1385-1392 (2012).
44. A. Pliss, X. Peng, L. Liu, A. Kuzmin, Y. Wang, J. Qu, Y. Li, P. N. Prasad, "Single cell assay for molecular diagnostics and medicine: Monitoring intracellular concentrations of macromolecules by two-photon fluorescence lifetime imaging," Theranostics 5, 919-930 (2015).

45. M. Bencina, "Illumination of the spatial order of intracellular $\mathrm{pH}$ by genetically encoded $\mathrm{pH}$-sensitive sensors," Sensors 13, 16736-16758 (2013).

46. T. Nakabayashi, S. Oshita, R. Sumikawa, F. Sun, M. Kinjo, N. Ohta, "pH dependence of the fluorescence lifetime of enhanced yellow fluorescent protein in solution and cells," J. Photochem. Photobiol. A 235, 65-71 (2012).

47. F. J. Schmitt, B. Thaa, C. Junghans, M. Vitali, M. Veit, T. Friedrich, "eGFP-pHsens as a highly sensitive fluorophore for cellular $\mathrm{pH}$ determination by fluorescence lifetime imaging microscopy (FLIM)," Biochem. Biophys. Acta 1837, 1581-1593 (2014).

48. T. Nakabayashi, H. P. Wang, M. Kinjo, N. Ohta, "Application of fluorescence lifetime imaging of enhanced green fluorescent protein to intracellular $\mathrm{pH}$ measurements," Photochem. Photobiol. Sci. 7, 668670 (2008).

49. S. Poea-Guyon, H. Pasquier, F. Merola, N. Morel, M. Erard, "The enhanced cyan fluorescent protein: A sensitive $\mathrm{pH}$ sensor for fluorescence lifetime imaging," Anal. Bioanal. Chem. 405, 3983-3987 (2013).

50. H. Ogawa, S. Inouye, F. I. Tsuji, K. Yasuda, K. Umesono, "Localization, trafficking, and temperaturedependence of the Aequorea green fluorescent protein in cultured vertebrate cells," Proc. Natl. Acad. Sci. USA 92, 11899-11903 (1995).

51. S. Kiyonaka, T. Kajimoto, R. Sakaguchi, D. Shinmi, M. Omatsu-Kanbe, H. Matsuura, H. Imamura, T. Yoshizaki, I. Hamachi, T. Morii, Y. Mori, "Genetically encoded fluorescent thermosensors visualize subcellular thermoregulation in living cells," Nat. Methods 10, 1232-1238 (2013).

52. M. Nakano, Y. Arai, I. Kotera, K. Okabe, Y. Kamei, T. Nagai, "Genetically encoded ratiometric fluorescent thermometer with wide range and rapid response," PLoS One 12, e0172344 (2017).

53. K. Deepankumar, S. P. Nadarajan, D. H. Bae, K. H. Baek, K. Y. Choi, H. Yun, "Temperature sensing using red fluorescent protein," Biotechnol. Bioprocess. Eng. 20, 67-72 (2015).

54. H. Itoh, S. Arai, T. Sudhaharan, S. C. Lee, Y. T. Chang, S. Ishiwata, M. Suzuki, E. B. Lane, "Direct organelle thermometry with fluorescence lifetime imaging microscopy in single myotubes," Chem. Commun. (Camb.) 52, 4458-4461 (2016). 
55. K. Okabe, N. Inada, C. Gota, Y. Harada, T. Funatsu, S. Uchiyama, "Intracellular temperature mapping with a fluorescent polymeric thermometer and fluorescence lifetime imaging microscopy," Nat. Commun. 3, 705 (2012).
56. B. Treanor, P. M. Lanigan, K. Suhling, T. Schreiber, I. Munro, M. A. Neil, D. Phillips, D. M. Davis, P. M. French, "Imaging fluorescence lifetime heterogeneity applied to GFP-tagged MHC protein at an immunological synapse," J. Microsc. 217, 36-43 (2005). 